\title{
Volaemic resuscitation
}

in critical patients with severe haemorrhagic shock. Complications due to impaired microvascular system and ischaemia/reperfusion syndrome: A Case Report

BY OVIDIU HOREA BEDREAG, OANA MARIA ROSU, ALEXANDRU FLORIN ROGOBETE, MIRELA SARANDAN, ALINA CARMEN CRADIGATI, MARIUS PAPURICA, BOGDAN TUNESCU, MIRCEA POPESCU, SILVIU DRAGUSANU, TUDOR COJOCARU, DOREL SANDESC

\section{Abstract}

Severe trauma is one of the most common causes of death all around the world. Complications of haemorrhagic shock, such as coagulopathy, hypothermia and metabolic acidosis, form the lethal triad in trauma. 
haemorrhagic shock - haemoglobin (Hb) $5.6 \mathrm{mg} / \mathrm{dL}$, arterial pressure (AP) $60 / 53 \mathrm{mmHg}$, heart beat (HB) $140 \mathrm{bpm}$, hypothermia $\left(35^{\circ} \mathrm{C}\right)$, lactic acidosis $\mathrm{pH}$ 7.21, lactates (Lac) $3.9 \mathrm{mmol} / \mathrm{L}$, excess bases $\mathrm{BE}(\mathrm{E})-12.8$ $\mathrm{mmol} / \mathrm{L}$.

Volume resuscitation was started in the emergency department (ED), and continued in the operating room (OR). Fluid resuscitation was guided taking into account a number of factors, such as maintaining fluid and electrolyte balance, protecting the microcirculation, avoidance of ischaemia / reperfusion syndrome, minimization and inhibition of free radical synthesis, responsible for oxidative stress, by administration of high doses of antioxidants.

During Intensive Care Unit (ICU) admission, the patient developed severe left lower limb ischaemia. After clinical and laboratory investigations, it was decided to perform a femoral-popliteal by-pass. Failure of the by-pass resulted in amputation of the left lower limb. The patient was discharged from ICU after 27 days of intensive therapy and nursing.

In conclusion, we can affirm that the adjustment of fluid management, as well as administration of substances with antioxidant properties, improved the clinical status of the patient and chance of survival.

Key words: haemorrhagic shock, volaemic resuscitation, ischaemia, reperfusion, oxidative stress

\section{Introduction}

Severe traumas are always coupled with severe injuries of the micro- and macro- vascular systems. Pre-existent vascular pathologies associated with trauma significantly worsen the clinical picture of the patient due to specific pathological consequences: free radical biosynthesis and oxidative stress (OS) and mitochondrial dysfunction. The magnitude of the systemic inflammatory response syndrome (SIRS) is connected to organ injuries: pulmonary, liver and kidney damage, intestinal injuries and brain injury. (1-3) In this case, OS and systemic inflammation, caused directly by haemorrhagic shock and the ischaemia/reperfusion 
syndrome, had a significant contribution to the development of multiple organ dysfunction syndrome (MODS). (4-6)

\section{Case presentation}

We are presenting the case of a 57 year old male, , height $180 \mathrm{~cm}$, weight $59 \mathrm{~kg}$, blood type A (II) Rh positive, admitted to the Emergency Department (ED) of the Emergency County Hospital "Pius Brinzeu" Timisoara, Romania, with multiple injuries. He sustained an open fracture of the right femur, thoracic injuries: fracture of the right axillary rib arch VI and VII, tension pneumothorax, and spinal cord injuries: T12 vertebral fracture, caused by a working accident (explosion of an underpressure tank).

Clinically, the patient presented with haemorrhagic shock, heart beat (HB) $140 \mathrm{bpm}$; arterial pressure (AP) 6o/53 mmHg; tachypnoea; $\mathrm{SpO}_{2}$ 85\%; anuria; central temperature $35^{\circ} \mathrm{C}$. Laboratory results showed: haemoglobin (Hb) $5.6 \mathrm{mg} / \mathrm{dL}$, haematocrit (Hct) $16 \%$, creatine kinase (CK) 1315 U/L, CK MB 89 U/L and lactate dehydrogenase (LDH) 1425 $\mathrm{U} / \mathrm{L}$. Arterial blood gases (ABGs) : $\mathrm{pH} 7.21 ; \mathrm{paCO}_{2} 39.8 \mathrm{mmHg} ; \mathrm{paO}_{2}$ $78.9 \mathrm{mmHg} ; \mathrm{HCO}_{3}{ }^{-} 13.4 \mathrm{mmol} / \mathrm{L} ; \mathrm{BE}(\mathrm{B})-12.8 \mathrm{mmol} / \mathrm{L} ; \mathrm{Na}^{+} 145.3$ $\mathrm{mmol} / \mathrm{L} ; \mathrm{K}^{+} 3.26 \mathrm{mmol} / \mathrm{L}$; glucose (Glu) $178 \mathrm{mg} / \mathrm{dL}$; lactate (Lac) 3.9 $\mathrm{mmol} / \mathrm{L}$. The patient developed severe anaemia ( $\mathrm{Hb} 4.7 \mathrm{mg} / \mathrm{dL}$ ), thrombocytopaenia (thrombocytes $62 \times 10^{3} / \mathrm{uI}$ ), post-traumatic coagulopathy (prothrombin time 14.9 s, prothrombin activity $68 \%$, fibrinogen $399 \mathrm{mg} / \mathrm{dL}$, international normalized ratio 1.78), lactic acidosis (Lac $5.9 \mathrm{mmol} / \mathrm{L}$ ) and rhabdomyolysis syndrome (CK $3100 \mathrm{U} / \mathrm{L}$, CK MB 102 U/L, LDH 1485 U/L).

The patient was brought to the operating room (OR) for stabilization with Ex-Fix of his left femoral fracture. On admission to the OR, the patient's condition deteriorated further: HB 135 bpm, AP 64/31 mmHg, Hb $4.2 \mathrm{mg}$ / dL, thrombocytes 52 x 103 / IU, Lac $6.3 \mathrm{mmol}$ / L. Volume resuscitation during surgery was optimized according to haemodynamic parameters (table 1). Moreover, to maintain haemodynamic status, administration of vasopressors was required (noradrenaline) and also factor VII 
(NovoSeven). Forty-eight hours post-surgery, the patient developed left lower limb ischaemia despite thromboprophylaxis with $40 \mathrm{mg}$ enoxaparine daily, started 24 hours after trauma. After 36 hours of ischaemia, a left femoral-popliteal bypass was performed together with decompressive fasciotomy at the calf level ( $4^{\text {th }}$ day).

On the $3^{\text {rd }}$ day, the patient was tracheostomised. The femoral-popliteal bypass was ineffective, and 6 days after admission, the patient developed septic shock. The clinical status of the patient progressively worsened, and after 8 days, the left lower limb was amputated. Antioxidative therapy was started as follows: Vitamin C (3000 $\mathrm{mg} /$ day for 5 days), Vitamin B1 (305 mg/day for 5 days), Vitamin E (6oo mg in the first day, and $300 \mathrm{mg} /$ day for another 5 days) and N-acetylcysteine (100 mg / kg / day for 7 days).

The patient's condition started to improve, allowing ventilator weaning after 20 days of mechanical ventilation, and tracheostomy decannulation after 22 days. The patient was discharged from the Intensive Care Unit (ICU) on day 27, and was transferred to the Traumatology Clinic.

\section{Discussion}

The most severe complications induced by trauma are represented by haemorrhagic shock and its particularities: hypothermia, hydroelectric imbalance, tissue hypoxia and dysfunctions in the microvascular system due to blood centralization. (7-10) In our case, volaemic resuscitation was performed by taking into account a series of criteria: maintaining volaemic equilibrium, maintaining electrolyte equilibrium, maintaining mean arterial pressure (mean arterial pressure (MAP) $>70 \mathrm{mmHg}$ ), protecting the microcirculation, avoidance of ischaemia/reperfusion syndrome, minimization and inhibition of free radical synthesis responsible for oxidative stress (figure 1).

The correlation between massive haemorrhagic shock, severe trauma, SIRS and lactic acidosis leads to severe coagulopathy - bleeding in the first stage and hypercoagulation later. (5-10) A progressive increase of lactate and a decrease of $\mathrm{pH}$ suggest anaerobic metabolism and 
mitochondrial dysfunction, which is usually followed by irreversible cell apoptosis, unless aggressive therapy is applied. . Microcirculation and tissue oxygenation were severely compromised in our case.

After 8 days it was decided that the left lower limb needed to be amputated. During surgery there were no major complications registered. The patient received substances with anti-oxidative properties to avoid excessive production of free radicals. The literature presents numerous studies regarding the action of oxidative stress on microcirculation. Moreover, several studies report improvement in outcomes after the implementation of antioxidant therapy. (10) In our case, the clinical status of the patient and the fast remission of inflammation can be correlated with the antioxidative treatment. After 18 days of hospitalization, when IL-6 was low, transpedicular fixation of the T12 thoracic vertebral fracture was performed under general anaesthesia. During and after surgery, the biochemical parameters remained constant, and no major biological imbalance was registered.

\section{Conclusion}

Our patient represents a complex case of trauma and haemorrhagic shock that required complex pharmacological therapy and surgical management. Finally, we can say that optimizing fluid resuscitation according to haemodynamic parameters represent the main goal in the management of haemorrhagic shock. Also, the combination of antioxidant therapy, with conventional intensive therapy, resulted in increased chances of survival by minimizing the inflammatory syndrome and its side effects.

\section{Acknowledgements}

The authors thank Ms. Corina Maria Dumbuleu MD, for correcting the article in English.

\section{References}


1. Arajibani MK, Ashemi MH, Ontazerifar FM. Effect of Vitamin E and C Supplements on Antioxidant Defense System in Cardiovascular Disease Patients in Zahedan. Southeast Iran 2010;436-40.

2. Yu G, Chi X, Hei Z, Shen N, Chen J, Zhang W, et al. Small volume resuscitation with $7.5 \%$ hypertonic saline, hydroxyethyl starch 130/0.4 solution and hypertonic sodium chloride hydroxyethyl starch 40 injection reduced lung injury in endotoxin shock rats: comparison with saline. Pulm Pharmacol Ther 2012;25:27-32.

3. Yu T-C, Yang F-L, Hsu B-G, Wu W-T, Chen S-C, Lee R-P, et al. Deleterious effects of aggressive rapid crystalloid resuscitation on treatment of hyperinflammatory response and lung injury induced by hemorrhage in aging rats. J Surg Res 2014;187:587-95.

4. Yamashita T, Sakamoto K, Yamanishi H, Totani N, Yamamoto J. Effect of a free radical scavenger on nitric oxide release in microvessels. Vascul Pharmacol 2013;58:134-9.

5. Huber S, Biberthaler P, Delhey P, Trentzsch H, Winter H, van Griensven $\mathrm{M}$, et al. Predictors of poor outcomes after significant chest trauma in multiply injured patients: a retrospective analysis from the German Trauma Registry (Trauma Register DGUß). Scand J Trauma Resusc Emerg Med 2014;22:52.

6. Venkata C, Kashyap R, Farmer JC, Afessa B. Thrombocytopenia in adult patients with sepsis: incidence, risk factors, and its association with clinical outcome. J Intensive Care 2013;1:9.

7. Chen G, You G, Wang Y, Lu M, Cheng W, Yang J, et al. Effects of synthetic colloids on oxidative stress and inflammatory response in hemorrhagic shock: comparison of hydroxyethyl starch 130/0.4, hydroxyethyl starch 200/0.5, and succinylated gelatin. Crit Care 2013;17:R141.

8. Curry N, Davis PW. What's new in resuscitation strategies for the patient with multiple trauma? Injury 2012;43:1021-8.

9. Nascimento B, Callum J, Rubenfeld G, Baptista J, Neto R, Lin Y, et al. Clinical review: Fresh frozen plasma in massive bleedings - more questions than answers. Crit Care 2014;14(1):202.

10. Tanaka K A, Mazzeffi M, Durila M. Role of prothrombin complex concentrate in perioperative coagulation therapy. J Intensive Care 2014;2:60. 
Figure 1. The dynamics of the mean arterial pressure (MAP) (A) and heart beat (HB) (B) during volaemic resuscitation. It can be observed that once blood products were administered, MAP increased significantly. At the same time, proper fluid resuscitation decreased the heart rate considerably. Post-surgical MAP and HB were maintained at a constant level, when using vasopressors and fluid therapy.

ED, emergency department; OR, operating room; ICU, intensive care unit.

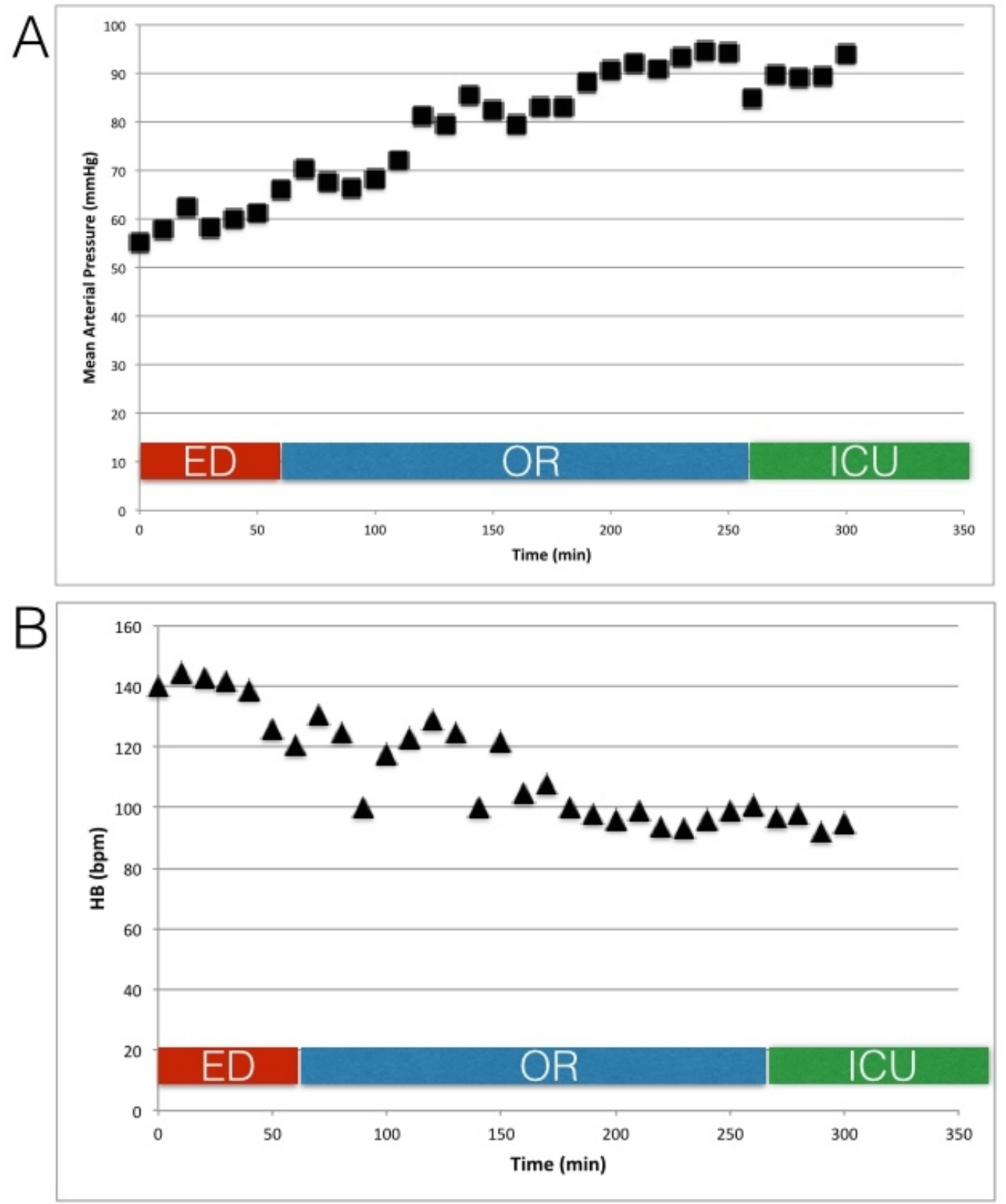

Table 1. Volaemic resuscitation scheme.

$$
1^{\text {st }} \mathrm{h} \quad 2^{\text {st }} \mathrm{h} \quad 3^{\mathrm{rd} h} \quad 12 \mathrm{~h} \quad 24 \mathrm{~h}
$$


(ED) (OR) (OR) (ICU) (ICU)

\begin{tabular}{llllll}
\hline FFP (unit) & 1 & 2 & 3 & 2 & 1 \\
\hline CP (unit) & 0 & 4 & 3 & 2 & 0 \\
\hline $\begin{array}{l}\text { PRBCs } \\
\text { (unit) }\end{array}$ & 0 & 0 & 9 & 1 & 1 \\
\hline $\mathrm{SC} \mathrm{(mL)}$ & 1500 & 500 & 0 & 0 & 0 \\
\hline
\end{tabular}

$\mathrm{CP}$, platelet concentrate; $\mathrm{ED}$, emergency department; FFP, fresh frozen plasma; ICU, intensive care unit; OR, operating room; PRBCs, packed red blood cells; SC, colloidal solutions (hydroxyethyl starch 130/o.4).

Corresponding author:

Ovidiu Horea Bedreag

Clinic of Anaesthesia and Intensive Care

Emergency County Hospital "Pius Brinzeu” Timisoara

Bd. Iosif Bulbuca nr.10, Timisoara, Timis, Romania

E-mail: bedreag.ovidiu@umft.ro

Article printed from Signa Vitae: http://www.signavitae.com

URL to article: http://www.signavitae.com/2015/12/volaemicresuscitation-in-critical-patients-with-severe-haemorrhagicshock-complications-due-to-impaired-microvascular-systemand-ischaemiareperfusion-syndrome-a-case-report/

Copyright (C) 2015 Signa Vitae. All rights reserved. 